\title{
Airflow Limitation Appearing as Premature Cycling on Expiratory Flow Waveform
}

\section{Stefano Arrigoni Marocco ${ }^{1}$, Giacomo Bellani ${ }^{1,2^{\star}}$, Francesca Rabboni ${ }^{1}$ and Antonio Pesenti ${ }^{1,2}$}

${ }^{1}$ Department of Health Sciences, University of Milan-Bicocca, Monza, Italy

${ }^{2}$ Department of Anesthesia and Intensive Care, San Gerardo Hospital, Monza, Italy

\section{Short Communication}

Patient-ventilator asynchronies are a relevant concern during assisted mechanical ventilation as they can lead to an increased work of breathing and potentially to patient's harm [1]. It is then paramount to rapidly detect and correct them.

A patient-ventilator asynchrony is usually recognized as airway waveforms abnormalities on the ventilator screen in the context of altered respiratory mechanics.

Measuring the activity of respiratory muscles, by monitoring oesophageal pressure (Pes) or the electrical activity of the diaphragm (EAdi), is a great aid in recognizing and correcting an asynchrony before the onset of the clinical counterpart.

A sudden rise of the flow waveform during expiration, leading to a biphasic expiration, may suggest different types of asynchrony, namely a premature cycling (termination of a breath while the patient is still inhaling), or an ineffective effort [2]. These asynchronies may be corrected by adjusting the expiratory and/or inspiratory trigger.

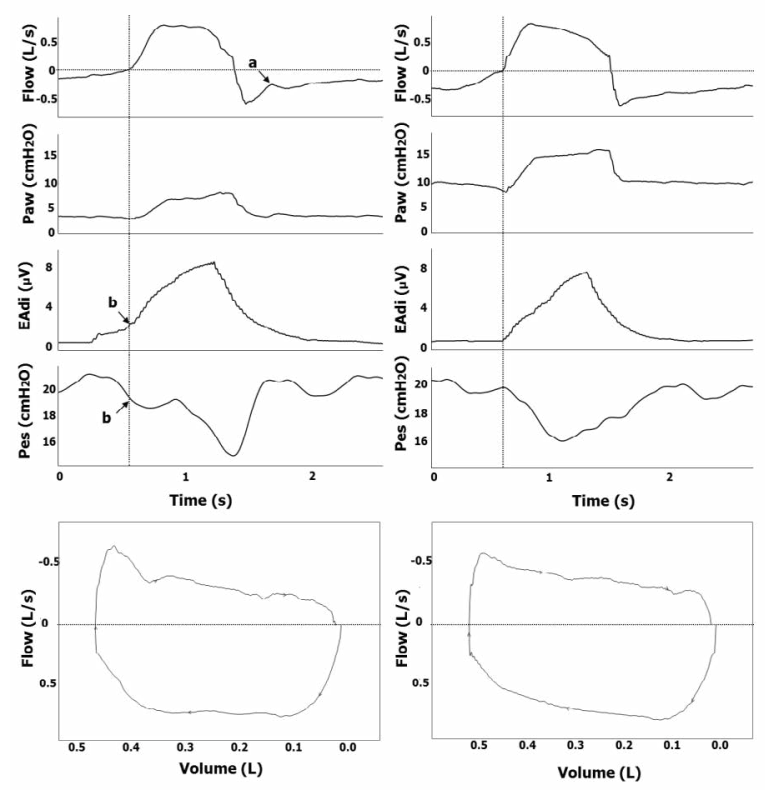

Figure 1: Airflow limitation appearing as premature cycling on expiratory flow waveform.
In a COPD patient we observed the aforementioned shape (left panel, arrow "a"), but some elements led us to a different diagnosis; indeed the flow drop was not due to a persisting inspiratory activity as shown by Pes and EAdi. However, the spirometry flow-volume plot revealed the concave expiratory shape, quite typical for airflow limitation.

Furthermore and in support of, those same breaths disclosed triggering delay both on the EAdi and Pes tracing (left panel, arrow "b"), disclosing the dissipation of the muscular pressure necessary to counterbalance intrinsic PEEP [3]. Our presumptive diagnosis of the observed asynchrony was expiratory flow limitation rather than a premature cycling or a missing effort (Figure 1).

Considering that, we neither adjusted the cycle off nor the trigger sensitivity; instead, we increased extrinsic PEEP obtaining the disappearance of the waveform abnormalities, eventually confirming our presumption.

\section{References}

1. Thille AW, Rodriguez P, Cabello B, Lellouche F, Brochard L (2006) Patientventilator asynchrony during assisted mechanical ventilation. Intensive Care Med 32: 1515-1522.

2. de Wit M (2011) Monitoring of patient-ventilator interaction at the bedside. Respir Care 56: 61-72.

3. Bellani G, Coppadoro A, Patroniti N, Turella M, Arrigoni Marocco S, et al. (2014) Clinical assessment of auto-positive end-expiratory pressure by diaphragmatic electrical activity during pressure support and neurally adjusted ventilatory assist. Anesthesiology 121: 563-571.
${ }^{*}$ Corresponding author: Giacomo Bellani, Department of Health Sciences, University of Milan-Bicocca, Monza, Italy, Tel: +39 (0)39 2333293; E-mail: giacomo.bellani1@unimib.it

Received Dec 30, 2014; Accepted July 15, 2015; Published July 20, 2015

Citation: Marocco SA, Bellani G, Rabboni F, Pesenti A (2015) Airflow Limitation Appearing as Premature Cycling on Expiratory Flow Waveform. J Pulm Respir Med 5: 272. doi:10.4172/2161-105X.1000272

Copyright: (c) 2015 Marocco SA, et al. This is an open-access article distributed under the terms of the Creative Commons Attribution License, which permits unrestricted use, distribution, and reproduction in any medium, provided the original author and source are credited. 